\title{
Fixed points of Lyapunov integral operators and Gibbs measures
}

\section{F. H. Haydarov ${ }^{1}$}

Received: 13 September 2017 / Accepted: 6 February 2018 / Published online: 13 February 2018 (C) The Author(s) 2018. This article is an open access publication

\begin{abstract}
In this paper we shall consider the connections between Lyapunov integral operators and Gibbs measures for models with four competing interactions and uncountable (i.e. $[0,1]$ ) set of spin values on a Cayley tree. We prove the existence of fixed points of Lyapunov integral operators and give a condition of uniqueness of a fixed point.
\end{abstract}

Keywords Cayley tree · Gibbs measures · Lyapunov integral operator · Fixed point

Mathematics Subject Classification Primary 82B05 - 82B20; Secondary 60K35

\section{Preliminaries}

A Cayley tree $\Gamma^{k}=(V, L)$ of order $k \in \mathbb{N}$ is an infinite homogeneous tree, i.e., a graph without cycles, with exactly $k+1$ edges incident to each vertices. Here $V$ is the set of vertices and $L$ that of edges (arcs). Two vertices $x$ and $y$ are called nearest neighbors if there exists an edge $l \in L$ connecting them. We will use the notation $l=\langle x, y\rangle$. The distance $d(x, y), x, y \in V$ on the Cayley tree is defined by the formula

$$
\begin{aligned}
& d(x, y)=\min \left\{d \mid x=x_{0}, x_{1}, \ldots, x_{d-1}, x_{d}=y \in V\right. \text { such that the pairs } \\
& \left.\left\langle x_{0}, x_{1}\right\rangle, \ldots,\left\langle x_{d-1}, x_{d}\right\rangle \text { are neighboring vertices }\right\}
\end{aligned}
$$

\footnotetext{
$凶 \quad$ F. H. Haydarov

haydarov_imc@mail.ru

1 National University of Uzbekistan, Tashkent, Uzbekistan
} 
Let $x^{0} \in V$ be a fixed and we set

$$
\begin{aligned}
& W_{n}=\left\{x \in V \mid d\left(x, x^{0}\right)=n\right\}, \quad V_{n}=\left\{x \in V \mid d\left(x, x^{0}\right) \leq n\right\}, \\
& L_{n}=\left\{l=\langle x, y\rangle \in L \mid x, y \in V_{n}\right\},
\end{aligned}
$$

The set of the direct successors of $x$ is denoted by $S(x)$, i.e.

$$
S(x)=\left\{y \in W_{n+1} \mid d(x, y)=1\right\}, x \in W_{n} .
$$

We observe that for any vertex $x \neq x^{0}, x$ has $k$ direct successors and $x^{0}$ has $k+1$. The vertices $x$ and $y$ are called second neighbor which is denoted by $\rangle x, y<$, if there exist a vertex $z \in V$ such that $x, z$ and $y, z$ are nearest neighbors. We will consider only second neighbors $\rangle x, y\left\langle\right.$, for which there exist $n$ such that $x, y \in W_{n}$. Three vertices $x, y$ and $z$ are called a triple of neighbors and they are denoted by $\langle x, y, z\rangle$, if $\langle x, y\rangle,\langle y, z\rangle$ are nearest neighbors and $x, z \in W_{n}, y \in W_{n-1}$, for some $n \in \mathbb{N}$.

Now we consider models with four competing interactions where the spin takes values in the set $[0,1]$. For some set $A \subset V$ an arbitrary function $\sigma_{A}: A \rightarrow[0,1]$ is called a configuration and the set of all configurations on $A$ we denote by $\Omega_{A}=$ $[0,1]^{A}$. Let $\sigma(\cdot)$ belong to $\Omega_{V}=\Omega$ and $\xi_{1}:(t, u, v) \in[0,1]^{3} \rightarrow \xi_{1}(t, u, v) \in R$, $\xi_{i}:(u, v) \in[0,1]^{2} \rightarrow \xi_{i}(u, v) \in R, i \in\{2,3\}$ are given bounded, measurable functions. Then we consider the model with four competing interactions on the Cayley tree which is defined by following Hamiltonian

$$
\begin{aligned}
& H(\sigma)=-J_{3} \sum_{\langle x, y, z\rangle} \xi_{1}(\sigma(x), \sigma(y), \sigma(z))-J \sum_{\langle x, y\langle} \xi_{2}(\sigma(x), \sigma(z)) \\
& -J_{1} \sum_{\langle x, y\rangle} \xi_{3}(\sigma(x), \sigma(y))-\alpha \sum_{x \in V} \sigma(x),
\end{aligned}
$$

where the sum in the first term ranges all triples of neighbors, the second sum ranges all second neighbors, the third sum ranges all nearest neighbors and $J, J_{1}, J_{3}, \alpha \in R \backslash\{0\}$. Let $h:(t, x) \in[0,1] \times V \backslash\left\{x^{0}\right\} \rightarrow h_{t, x} \in \mathbb{R}$ and $\left|h_{t, x}\right|<C$ where $x^{0}$ is a root of Cayley tree and $C$ is a constant which does not depend on $t$. For some $n \in \mathbb{N}$, $\sigma_{n}: x \in V_{n} \mapsto \sigma(x)$ and $Z_{n}$ is the corresponding partition function we consider the probability distribution $\mu^{(n)}$ on $\Omega_{V_{n}}$ defined by

$$
\begin{aligned}
\mu^{(n)}\left(\sigma_{n}\right) & =Z_{n}^{-1} \exp \left(-\beta H\left(\sigma_{n}\right)+\sum_{x \in W_{n}} h_{\sigma(x), x}\right), \\
Z_{n} & =\int \ldots \int_{\Omega_{V_{n-1}(p)}} \exp \left(-\beta H\left(\widetilde{\sigma}_{n}\right)+\sum_{x \in W_{n}} h_{\widetilde{\sigma}(x), x}\right) \lambda_{V_{n-1}}^{(p)}\left(d \widetilde{\sigma}_{n}\right),
\end{aligned}
$$


where for a set $A \subset V$ we denoted

$$
\underbrace{\Omega_{A} \times \Omega_{A} \times \ldots \times \Omega_{A}}_{3 \cdot 2^{p-1}}=\Omega_{A}^{(p)}, \underbrace{\lambda_{A} \times \lambda_{A} \times \ldots \times \lambda_{A}}_{3 \cdot 2^{p-1}}=\lambda_{A}^{(p)}, n, p \in \mathbb{N},
$$

Let $\sigma_{n-1} \in \Omega_{V_{n-1}}$ and $\sigma_{n-1} \vee \omega_{n} \in \Omega_{V_{n}}$ is the concatenation of $\sigma_{n-1}$ and $\omega_{n}$. For $n \in \mathbb{N}$ we say that the probability distributions $\mu^{(n)}$ are compatible if $\mu^{(n)}$ satisfies the following condition:

$$
\iint_{\Omega_{W_{n}} \times \Omega_{W_{n}}} \mu^{(n)}\left(\sigma_{n-1} \vee \omega_{n}\right)\left(\lambda_{W_{n}} \times \lambda_{W_{n}}\right)\left(d \omega_{n}\right)=\mu^{(n-1)}\left(\sigma_{n-1}\right) .
$$

By Kolmogorov's extension theorem there exists a unique measure $\mu$ on $\Omega_{V}$ such that, for any $n$ and $\sigma_{n} \in \Omega_{V_{n}}, \mu\left(\left\{\left.\sigma\right|_{V_{n}}=\sigma_{n}\right\}\right)=\mu^{(n)}\left(\sigma_{n}\right)$. The measure $\mu$ is called splitting Gibbs measure corresponding to Hamiltonian (1.1) and function $x \mapsto h_{x}=$ $\left\{h_{x, t}\right\}, x \neq x^{0}$ (see $\left.[1,2,5,7]\right)$.

Denote

$$
\begin{aligned}
K(t, u, v)= & \exp \left\{J_{3} \beta \xi_{1}(t, u, v)+J \beta \xi_{2}(u, v)+J_{1} \beta\left(\xi_{3}(t, u)+\xi_{3}(t, v)\right)\right. \\
& +\alpha \beta(u+v)\},
\end{aligned}
$$

and

$$
f(t, x)=\exp \left(h_{t, x}-h_{0, x}\right), \quad(t, u, v) \in[0,1]^{3}, x \in V \backslash\left\{x^{0}\right\} .
$$

The following statement describes conditions on $h_{x}$ guaranteeing compatibility of the corresponding distributions $\mu^{(n)}\left(\sigma_{n}\right)$.

Proposition 1.1 [6] Let $k=2$. The measure $\mu^{(n)}\left(\sigma_{n}\right), n=1,2, \ldots$ satisfies the consistency condition (1.4) iff for any $x \in V \backslash\left\{x^{0}\right\}$ the following equation holds:

$$
f(t, x)=\frac{\int_{0}^{1} \int_{0}^{1} K(t, u, v) f(u, y) f(v, z) d u d v}{\int_{0}^{1} \int_{0}^{1} K(0, u, v) f(u, y) f(v, z) d u d v},
$$

where $S(x)=\{y, z\}$.

\section{Existence of a fixed point of the operator $\mathcal{L}$}

Now we prove that there exist at least one fixed point of Lyapunov integral equation, namely there is a splitting Gibbs measure corresponding to Hamiltonian (1.1).

Proposition 2.1 Let $k=2, J_{3}=J=\alpha=0$ and $J_{1} \neq 0$. Then (1.6) is equivalent to

$$
f(t, x)=\prod_{y \in S(x)} \frac{\int_{0}^{1} \exp \left\{J_{1} \beta \xi_{3}(t, u)\right\} f(u, y) d u}{\int_{0}^{1} \exp \left\{J_{1} \beta \xi_{3}(0, u)\right\} f(u, y) d u},
$$


where $f(t, x)=\exp \left(h_{t, x}-h_{0, x}\right), t \in[0,1], x \in V$.

Proof For $J_{3}=J=\alpha=0$ and $J_{1} \neq 0$ one gets $K(t, u, v)=\exp \left\{J_{1} \beta\left(\xi_{3}(u, t)\right.\right.$ $\left.\left.+\xi_{3}(v, t)\right)\right\}$. Then (1.6) can be written as

$$
\begin{gathered}
f(t, x)=\frac{\int_{0}^{1} \int_{0}^{1} \exp \left\{J_{1} \beta\left(\xi_{3}(t, u)+\xi_{3}(t, v)\right)\right\} f(u, y) f(v, z) d u d v}{\int_{0}^{1} \int_{0}^{1} \exp \left\{J_{1} \beta\left(\xi_{3}(0, u)+\xi_{3}(0, v)\right)\right\} f(u, y) f(v, z) d u d v} \\
=\frac{\int_{0}^{1} \exp \left\{J_{1} \beta \xi_{3}(t, u)\right\} f(u, y) d u \cdot \int_{0}^{1} \exp \left\{J_{1} \beta \xi_{3}(t, v)\right\} f(v, z) d v}{\int_{0}^{1} \exp \left\{J_{1} \beta \xi_{3}(0, u)\right\} f(u, y) d u \cdot \int_{0}^{1} \exp \left\{J_{1} \beta \xi_{3}(0, v)\right\} f(v, z) d v} .
\end{gathered}
$$

Since $\rangle y, z\langle=S(x)$ Eq. (2.2) is equivalent to (2.1).

Now we consider the model (1.1) in the class of translational-invariant functions $f(t, x)$ i.e $f(t, x)=f(t)$, for any $x \in V$. For such functions Eq. (1.1) can be written as

$$
f(t)=\frac{\int_{0}^{1} \int_{0}^{1} K(t, u, v) f(u) f(v) d u d v}{\int_{0}^{1} \int_{0}^{1} K(0, u, v) f(u) f(v) d u d v},
$$

where $K(t, u, v)=\exp \left\{J_{3} \beta \xi_{1}(t, u, v)+J \beta \xi_{2}(u, v)+J_{1} \beta\left(\xi_{3}(t, u)+\xi_{3}(t, v)\right)\right.$ $+\alpha \beta(u+v)\}, f(t)>0, t, u \in[0,1]$.

We shall find positive continuous solutions to (2.3) i.e. such that $f \in C^{+}[0,1]=$ $\{f \in C[0,1]: f(x)>0\}$.

Define a nonlinear operator $H$ on the cone of positive continuous functions on $[0,1]$ :

$$
(H f)(t)=\frac{\int_{0}^{1} \int_{0}^{1} K(t, s, u) f(s) f(u) d s d u}{\int_{0}^{1} \int_{0}^{1} K(0, s, u) f(s) f(u) d s d u} .
$$

We'll study the existence of positive fixed points for the nonlinear operator $H$ (i.e., solutions of the Eq. (2.3)).

We define the Lyapunov integral operator $\mathcal{L}$ on $C[0,1]$ by the equality (see [3])

$$
\mathcal{L} f(t)=\int_{0}^{1} K(t, s, u) f(s) f(u) d s d u .
$$

Put

$$
\mathcal{M}_{0}=\left\{f \in C^{+}[0,1]: f(0)=1\right\}
$$

Lemma 2.2 The equation $H f=f$ has a nontrivial positive solution iff the Lyapunov equation $\mathcal{L} g=g$ has a nontrivial positive solution.

Proof At first we shall prove that the equation

$$
H f=f, \quad f \in C_{0}^{+}[0,1]
$$


has a positive solution iff the Lyapunov equation

$$
\mathcal{L} g=\lambda g, \quad g \in C^{+}[0,1]
$$

has a positive solution in $\mathcal{M}_{0}$ for some $\lambda>0$.

Let $\lambda_{0}$ be a positive eigenvalue of the Lyapunov operator $\mathcal{L}$. Then there exists $f_{0} \in C_{0}^{+}[0,1]$ such that $\mathcal{L} f_{0}=\lambda_{0} f_{0}$. Take $\lambda \in(0,+\infty), \lambda \neq \lambda_{0}$. Define the function $h_{0}(t) \in C_{0}^{+}[0,1]$ by $h_{0}(t)=\frac{\lambda}{\lambda_{0}} f_{0}(t), \quad t \in[0,1]$. Then $\mathcal{L} h_{0}=\lambda h_{0}$, i.e., the number $\lambda$ is an eigenvalue of Lyapunov operator $\mathcal{L}$ corresponding the eigenfunction $h_{0}(t)$. It's easy to check that if the number $\lambda_{0}>0$ is an eigenvalue of the operator $\mathcal{L}$, then an arbitrary positive number is eigenvalue of the operator $\mathcal{L}$. Now we shall prove the lemma. Let Eq. (2.4) holds then the function $\frac{1}{\lambda} g(t)$ be a fixed point of the operator $\mathcal{L}$. Analogously, since $H$ is non-linear operator we can correspond to the fixed point if there exist any eigenvector.

Proposition 2.3 The equation

$$
\mathcal{L} f=\lambda f, \quad \lambda>0
$$

has at least one solution in $C_{0}^{+}[0,1]$.

Proof Clearly, that the Lyapunov operator $\mathcal{L}$ is a compact on the cone $C^{+}[0,1]$. By the other hand we have

$$
\mathcal{L} f(t) \geq m\left(\int_{0}^{1} f(s) d s\right)^{2}
$$

for all $f \in C^{+}[0,1]$, where $m=\min K(t, s, u)>0$.

Put $\Gamma=\{f:\|f\|=r, \quad f \in C[0,1]\}$. We define the set $\Gamma_{+}$by

$$
\Gamma_{+}=\Gamma \cap C^{+}[0,1]
$$

Then we obtain

$$
\inf _{f \in \Gamma_{+}}\|\mathcal{L} f\|>0
$$

Then by Schauder's theorem (see [4], p.20) there exists a number $\lambda_{0}>0$ and a function $f_{0} \in \Gamma_{+}$such that, $\mathcal{L} f_{0}=\lambda_{0} f_{0}$.

Denote by $N_{\text {fix.p }}(H)$ and $N_{\text {fix.p }}(\mathcal{L})$ the set of positive numbers of nontrivial positive fixed points of the operators $H$ and $L$, respectively. By Lemma 2.2 and Proposition 2.3 we can conclude that:

Proposition 2.4 (a) The Eq. (2.4) has at least one solution in $C_{0}^{+}[0,1]$.

(b) The equality $N_{\text {fix.p }}(H)=N_{\text {fix.p }}(\mathcal{L})$ is hold.

From Propositions 1.1 and 2.4 we get the following theorem.

Theorem 2.5 The set of splitting Gibbs measures corresponding to Hamiltonian (1.1) is non-empty. 


\section{The uniqueness of fixed point of the operator $\mathcal{L}$}

In this section we shall give a condition of the uniqueness of fixed point of the operator $\mathcal{L}$.

Theorem 3.1 Let the kernel $K(t, u, v)$ satisfies the condition

$$
\max _{(t, u, v) \in[0,1]^{3}} K(t, u, v)<c \min _{(t, u, v) \in[0,1]^{3}} K(t, u, v), c \in\left(1, \frac{1}{2} \sqrt{\sqrt{17}+1}\right) .
$$

Then the operator $\mathcal{L}$ has the unique fixed point in $C_{0}^{+}[0,1]$.

Proof Let $\max _{(t, u, v) \in[0,1]^{3}} K(t, u, v)=\mathcal{K}$ and $\min _{(t, u, v) \in[0,1]^{3}} K(t, u, v)=k$. At first we shall prove that if $g \in C_{0}^{+}[0,1]$ is a solution of the equation $\mathcal{L} f=f$ then $g \in \mathcal{G}$ where

$$
\mathcal{G}=\left\{f \in C[0,1]: \frac{k}{\mathcal{K}^{2}} \leq f(t) \leq \frac{\mathcal{K}}{k^{2}}\right\}
$$

Let $s \in \mathcal{L}\left(C^{+}[0,1]\right)$ be an arbitrary function. Then there exists a function $h \in C^{+}[0,1]$ such that $s=\mathcal{L} h$. Since $s$ is continuous on $[0,1]$, there exists $t_{1}, t_{2} \in[0,1]$ such that

$$
s_{\min }=\min _{t \in[0,1]} s(t)=s\left(t_{1}\right)=(\mathcal{L} h)\left(t_{1}\right), \quad s_{\max }=\max _{t \in[0,1]} s(t)=s\left(t_{2}\right)=(\mathcal{L} h)\left(t_{2}\right) .
$$

Consequently we get

$$
s_{\min } \geq k \int_{0}^{1} \int_{0}^{1} h(u) h(v) d u d v \geq k \int_{0}^{1} \int_{0}^{1} \frac{K\left(t_{2}, u, v\right)}{\mathcal{K}} h(u) h(v) d u d v=\frac{k}{\mathcal{K}} s_{\max } .
$$

Since $g$ is a fixed point of the operator $\mathcal{L}$ we have $\|g\| \leq \mathcal{K}\|g\|^{2} \Rightarrow\|g\| \geq \frac{1}{\mathcal{K}}$.

From (3.2)

$$
g(t) \geq g_{\min }=\min _{t \in[0,1]} g(t) \geq \frac{k}{\mathcal{K}}\|g\| \Rightarrow g(t) \geq \frac{k}{\mathcal{K}^{2}}
$$

Similarly,

$$
g(t)=(\mathcal{L} g)(t) \geq k \int_{0}^{1} \int_{0}^{1} g(u) g(v) d u d v \geq k g_{\min }^{2} \Rightarrow g_{\min } \leq \frac{1}{k} .
$$

Hence

$$
g(t) \leq g_{\max } \leq \frac{\mathcal{K}}{k} g_{\min } \leq \frac{\mathcal{K}}{k^{2}} .
$$

Thus we have $g \in \mathcal{G}$. 
Now we show that $\mathcal{L}$ has the unique fixed point. By Proposition 2.4, $\mathcal{L} g=g$ has at least one solution. Assume that there are two solutions $g_{1} \in C_{0}^{+}[0,1]$ and $g_{2} \in C_{0}^{+}[0,1]$, i.e $\mathcal{L} g_{i}=g_{i}, i=1,2$.

Let a function $f \in C[0,1]$ changes its sign on $[0,1]$. Then it is easy to check that for every $a \in \mathbb{R}$ the following inequality holds: $\|f(t)-a\| \geq \frac{1}{2}\|f\|$.

Put $\xi(t)=g_{1}(t)-g_{2}(t)$. Since $\xi(t)$ changes its sign on $[0,1]$, we get

$$
\begin{aligned}
& \max _{t \in[0,1]}\left|\xi(t)-\left(\frac{k^{2}}{\mathcal{K}^{2}}+\frac{\mathcal{K}^{2}}{k^{2}}\right) \int_{0}^{1} \xi(s) d s\right| \geq \frac{1}{2}\|\xi\|, \\
& \xi(t)=2 \int_{0}^{1} \int_{0}^{1} K(t, u, v)\left(g_{1}(u) g_{1}(v)-g_{2}(u) g_{2}(v)\right) d u d v .
\end{aligned}
$$

The last equation can be written as

$$
\xi(t)=\int_{0}^{1} \int_{0}^{1} K(t, u, v) \eta(u, v)(|\xi(u)-\xi(v)|+\xi(u)+\xi(v)) d u d v
$$

where

$$
\min \left\{g_{1}(t), g_{2}(t)\right\} \leq \eta(u, v) \leq \max \left\{g_{1}(t), g_{2}(t)\right\}, t \in[0,1]
$$

Since $g_{i}(t) \in \mathcal{G}, i=\overline{1,2}$ we get $\frac{k}{\mathcal{K}^{2}} \leq \eta(u, v) \leq \frac{\mathcal{K}}{k^{2}},(u, v) \in[0,1]^{2}$. Hence

$$
\left|2 \cdot K(t, u, v) \eta(u, v)-\left(\frac{\mathcal{K}^{2}}{k^{2}}+\frac{k^{2}}{\mathcal{K}^{2}}\right)\right| \leq \frac{\mathcal{K}^{2}}{k^{2}}-\frac{k^{2}}{\mathcal{K}^{2}}
$$

Then

$$
\begin{aligned}
& \left|\xi(t)-\left(\frac{\mathcal{K}^{2}}{k^{2}}+\frac{k^{2}}{\mathcal{K}^{2}}\right) \int_{0}^{1} \int_{0}^{1}(|\xi(u)-\xi(v)|+\xi(u)+\xi(v)) d u d v\right| \\
& \leq\left(\frac{\mathcal{K}^{2}}{k^{2}}-\frac{k^{2}}{\mathcal{K}^{2}}\right)\|\xi\| .
\end{aligned}
$$

Assume the kernel $K(t, u, v)$ satisfies the condition (3.1). Then $\mathcal{K}^{4}-k^{4}<(\mathcal{K} k)^{2} \Rightarrow$ $\mathcal{K}<c k$ but it's contradict to the following: if $\xi \in C[0,1]$ changes its sign on $[0,1]$ then for every $a \in \mathbb{R}$ the following inequality holds $\|\xi-a\| \geq \frac{1}{2}\|\xi\|$. This completes the proof.

Theorem 3.2 Let $k \geq 2$. If the function $K(t, u, v)$ which defined in (1.5) satisfies the condition (3.1), then the model (1.1) has the unique-translational invariant Gibbs measure.

Acknowledgements Author thanks the referee for careful reading of the manuscript; in particular, for a number of comments which have improved the paper. 
Open Access This article is distributed under the terms of the Creative Commons Attribution 4.0 International License (http://creativecommons.org/licenses/by/4.0/), which permits unrestricted use, distribution, and reproduction in any medium, provided you give appropriate credit to the original author(s) and the source, provide a link to the Creative Commons license, and indicate if changes were made.

\section{References}

1. Eshkabilov, Y.K., Haydarov, F.H., Rozikov, U.A.: Non-uniqueness of Gibbs measure for models with uncountable set of spin values on a Cayley tree. J. Stat. Phys. 147, 779-794 (2012)

2. Eshkabilov, Y.K., Nodirov, S.D., Haydarov, F.H.: Positive fixed points of quadratic operators and Gibbs measures. Positivity (2016). https://doi.org/10.1007/s11117-015-0394-9

3. Krasnosel'ski, M.A.: Positive Solutions of Opertor Equations. Gos. Izd, Moscow (1969). (Russian)

4. Nirenberg, L.: Topics in nonlinear functional analysis. AMS, Courant Lecture Notes in Math, pp. 6 (2001)

5. Rozikov, U.A.: Gibbs Measures on a Cayley Trees. World Sci. Pub, Singapore (2013)

6. Rozikov, U.A., Haydarov, F.H.: Four competing interactions for models with an uncountable set of spin values on a Cayley tree. Theor. Math. Phys. 191(2), 748-761 (2017)

7. Spitzer, F.: Markov random fields on an infinite tree. Ann. Probab. 3, 387-398 (1975) 